\title{
Synchronous Phasors Monitoring System Application Possibilities
}

\author{
Andrew G. Kasembe, Zdenek Muller, Jan Svec \\ and Josef Tlusty \\ Deptartment of Electrical Power Engineering \\ Czech Technical University in Prague \\ Prague, Czech Republic \\ zdenek.muller@fel.cvut.cz
}

\author{
Viktor Valouch \\ Institute of Thermomechanics \\ Academy of Sciences of the Czech Republic \\ Prague, Czech Republic \\ valouch@it.cas.cz
}

\begin{abstract}
The paper deals with some tasks of advanced methods to achieve security and reliability of transmission power systems. There are discussed advanced principles and techniques in monitoring systems. The paper main part is devoted to possible applications of PMUs (Phasor Measurement Unit) and their integration into WAMPaC systems (Wide Area Monitoring, Protection and Control). Significant transmission system states were analyzed in several case studies. PMUs utilization for voltage monitoring is demonstrated for three cases - standard system state, high-load state and islanding. Here the attention is paid to the possibilities of power system observability improvement using measuring chain error elimination. Other possible applications in transmission systems include ampacity determination of transmission corridors, the calculation of line electrical parameters using synchrophasor measurement, state estimation issues and power system stability. The power system model was established for methods and algorithms verification purposes. The appropriate algorithms and their parameters were found using simulation outputs to create the local automatics, e.g. Automatic Power System Stabilizer (PSS).
\end{abstract}

Index Terms - Phasor Measurement Unit, Wide Area Monitoring System, Transmission System, Power System Stability, Extraordinary States, Black-out, Loop Flows

\section{INTRODUCTION}

The deregulation of the electric power industry, the demands for better energy quality and restrictions on the expansion of the transmission and distribution system have increased the complexity of the power system operation. While the power system is continuously exposed to different extraordinary states that range from small ones with little impact on operation to large ones with severe consequences, including blackouts, secure and reliable operation remains to be a fundamental requirement for a power network system. In addition to those facts mentioned above (which can be classified as long term and development planning factors), unpredicted events or states can stress even the best-planned system beyond the acceptable limits. Upon capturing and acquiring a precise knowledge, a power system disturbance is mitigated via protection and control actions, required to stop the power system degradation, restore the normal state and minimize the impact of disturbances.

\section{A. Phasor Measurement}

Monitoring of events in the power system itself provides a great deal of insight into the behaviour of the system. Most of these severe events with impact on the entire power system typically occur in or near the transmission network and are therefore best monitored at transmission system substations. This puts great demands on equipment and in general restricts the use of the data since it becomes property of the transmission system operator. An alternative is measurement at lower voltage levels.

Here, a point can be made that, the application of PMUs has been rising as one of the new tools to improve the power system monitoring and performance. A more complex system comprising of PMUs is usually referred as Wide Area Measurement System (WAMS). These systems are basically composed by PMUs connected to a Phasor Data Concentrator (PDC) and applications for monitoring and control of power system real time operation. [1], [2]

Only to mention, the following issues from power industry deregulation point of view have heighten need for more rigorous security assessment:

- Aging transmission and distribution infrastructures, usually means potential components failures

- Lack of new transmission and distribution facilities could mean or cause bottlenecks

- Increasing number of small and distributed generators brings uncertainty in dispatch patterns

- Cutbacks in system maintenance that means potential for failures such as flashovers to trees

- Dependence on information technology (telecom and computer systems), as result to this, failures may leave operators blind

- Expansion of interconnection network system may lead to exposure to cascading disturbances.

- Insufficient and/or improper generation/transmission resources which may result due to lack of integrated system planning

Some of the reasons for less than complete system reliability are: 
- Unpredicted changes in power systems which may differ dramatically from the forecasts

- A very large number of possible operating contingencies

- A combination of unusual and undesired events (for example, human error combined with heavy weather or other combination of events that would be considered very improbable in the earlier times of power system evolution

- Reliability design philosophy that is pushing the system close to the limits, imposed by both economic and environmental pressures

The most often disturbance aspects have required better tools for monitoring and control for power system in real time operation. Conventional operating guides derived by conducting off-line studies have become inadequate because number of possible conditions and contingencies becomes unmanageable as the system complexity increases, the exact system state never accurately captured by forecast, most study results never used and some conditions which usually cause problems have never studied off-line (such as N-1 event to N-3 event).

As a promising solution is an On-line security monitoring and assessment system which can be based for example on measurements from Phasor Measurements Units. This system provides a possibility for a network topology and operating conditions to be modelled in real time as seen by the operator in the control room. A variety of methods of monitoring, detecting and locating faults on power transmission and distribution system exist. Most of these methods utilize measurements from voltage and current transformers at substations or switching stations to perform their analyses. To gain knowledge about the disturbances and their impact, it is necessary to monitor the system at sufficiently many geographical locations using measurement equipments with sufficient bandwidth. The collected data must also be condensed into useful information and properly analyzed.

\section{B. Synchronous phasors}

Phasor represents information about the magnitude of measured quantity and about its rotation - angle with respect to reference vector. A synchronous phasor is characterized by a position - angle in the selected time point. A synchronous phasors set of electric quantities in the transmission network is denoted as a phasor snap of the system. [4]

- All phasors in one snap are obtained exactly in the same time

- The reference vector has zero angle

- Phasor is a complex number associated with the cosine wave

- Time dimension is already contained in the phasor snap

- Fundamentals for synchronous measurement and communication are described in IEEE standards.

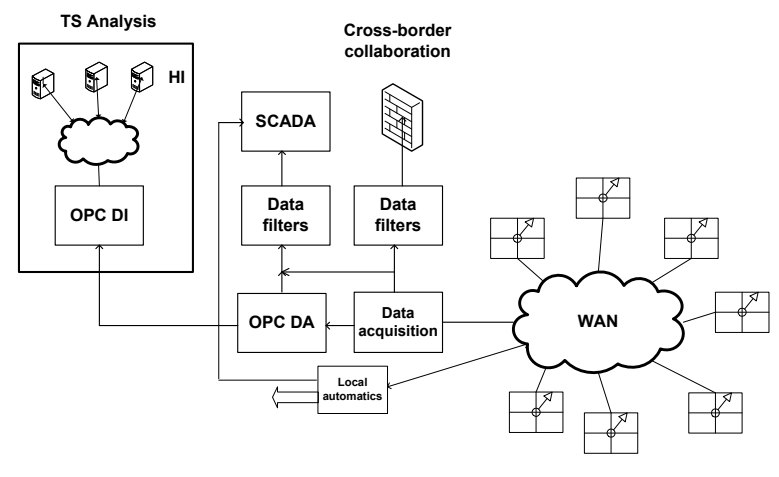

Fig. 1: Wide Area Monitoring System architecture

\section{CASE STUDY}

The system for synchronous phasor measurement in the electrical power system in the Czech Republic is in its early state. Measured data are stored and processed off-line. Terminals for the phasor measurement (PMU) are placed in switching stations. Selected busbars, lines and generator outlets are fitted with PMU. Measured data are transferred to the central phasor data concentrator (PDC) via reserved communication channels. Consequently, the data are processed and network phasor snaps are created from data measured in the same time point.

The network phasor snap is presented to the users in a suitable format and it is also continuously transferred to other applications for user processing. Examples of consequent applications can be e.g. dispatcher system, state estimation and network status calculation programs, network stability analysis etc. Archive data for analysis of network behavior are also available.

The Czech transmission system model was created in SW Mathematica for $400 \mathrm{kV}$ and $220 \mathrm{kV}$ voltage levels. There were monitored node voltages angles in ES substations. The most important corridor from the overloading viewpoint was "north-south". The maximal angles difference in a common state was $3.3^{\circ}$ between the corridor end substations. It is important to notice that this common state was for the highly loaded Czech ES but not affected by strong transit active power loop flows.

The next simulation describes a high active power flow through the Czech ES caused by an icreased production in wind farms in the north Germany resulting in loop flows through the Czech ES. The total transit in the most affected north-south corridor was increased by $2500 \mathrm{MW}$ against the common state. This power was transmitted from Germany and Poland to Austria and Slovakia. The maximal angles difference was increased to $36^{\circ}$ between the corridor end substations. Fig. 2 shows together the common state (black columns) and the extraordinary state (red columns). Several lines were loaded to their limits in the simulated state. 


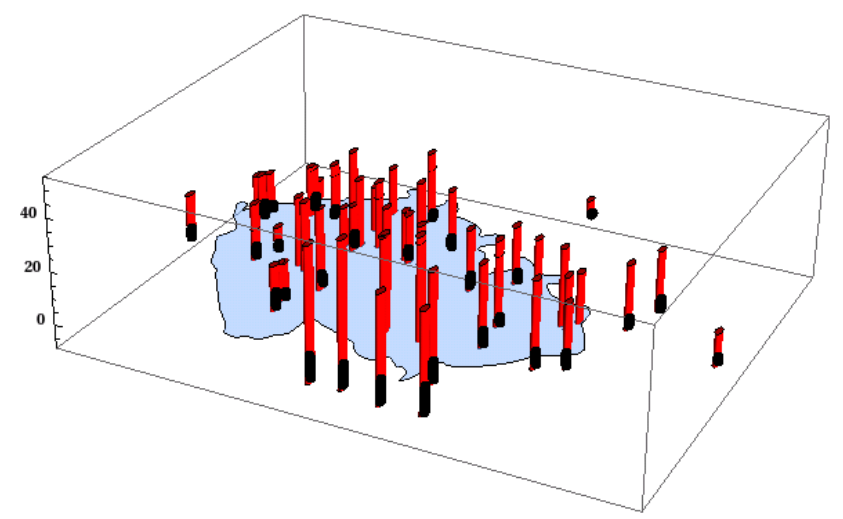

Fig. 2: Czech ES simulation results (phase shifts to the reference node) common state (black columns) and extraordinary state (red columns), phase shifts in deg to the reference node

The other section is dedicated to the description of the fault event that occurred in the Czech transmission system and was captured by PMUs which are installed in some power system substations. There was a very hot day almost with tropical temperatures when the event occurred. The actual day energy consumption was much higher than its forecasts. This increase of energy consumption was registered not only in the CR but also in other parts of Europe. Some transmission lines in the $\mathrm{CR}$ were under a planned maintenance (i.e. were disconnected). A combination of unusual events (line disconnections due to short circuits together with the decision of the dispatching centre to trip one line because of overload) triggered other cascading disturbances. [3]

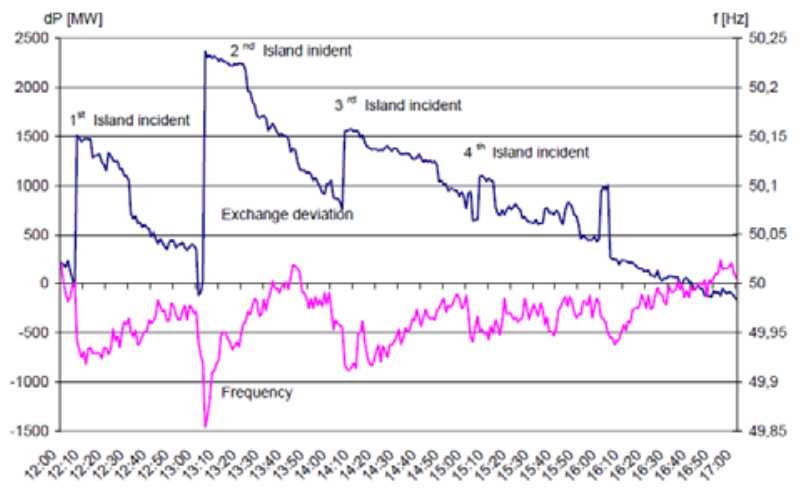

Fig. 3: PMUs measurement in the moments of island operation occurrence

It is assumed that this was related to the oscillation phenomenon which was registered at this moment. Because of these outages the Czech system broke into two islanding operations. One part had an excess of power production over its consumption, frequency of this part exceeded the predefined upper limit, i.e. $50.2 \mathrm{~Hz}$. In contrary, the other part had a power deficiency of about $1500 \mathrm{MW}$ and the frequency went down to the lower limit $49.8 \mathrm{~Hz}$. The resulted power unbalance affected the CR planned power exchange. The described phenomenon which resulted into islanding operation was precisely captured by PMUs installed in the transmission system. Frequency deviation which was computed from the PMU measurements indicates how the system frequency gradually changed before the whole system broke into islands (see Fig. 3). This picture demonstrates a very good visibility of a dynamic process in the transmission system.

\section{CONCLUSION}

The paper deals with using of advanced progressive tools for increasing reliability, safety and stability of electrical power systems. A using of phasor measurement units and mathematical simulations showed that these tools can identify dangerous power system states before coming collapse. When comparing the measured voltage angles differences for common and extraordinary state we can come to a conclusion for PMUs installation and utilization. Some critical extraordinary states can be developed gradually and their first stages can be close to common states and they can occur in more transmission systems in terms of European interconnected systems. The presented data shows that some critical states (in their early stages) can be under an observability level when using PMUs only in one transmission system. Therefore the real efficient tool will be obtained only if the monitoring system covers a wide area as European interconnected grids. This must be also followed by an online data exchange platform and sophisticated mathematical pattern recognition methods.

\section{ACKNOWLEDGMENT}

The financial support of the Ministry of Education, Youth and Sports of the Czech Republic, through grant number MSM 6840770017 , is highly acknowledged.

\section{REFERENCES}

[1] Depablos, J.; Centeno, V.; Phadke, A.G.; Ingram, M.; Comparative testing of synchronized phasor measurement units. Power Engineering Society General Meeting, 2004. IEEE, 6-10 June 2004 Page(s):948 - 954 Vol.1.

[2] Phadke, A.G.; Kasztenny, B.; Synchronized Phasor and Frequency Measurement Under Transient Conditions. Power Delivery, IEEE Transactions on, Volume 24, Issue 1, Jan. 2009 Page(s):89 - 95.

[3] Tlustý, J. - Kasembe, A. - Müller, Z. - Švec, J. Sýkora, T. - et al.: The Monitoring of Power System Events on Transmission and Distribution Level by the use of Phasor Measurement Units (PMU). In 20th International Conference and Exhibition on Electricity Distribution [CD-ROM]. Stevenage: IET Publishing Group, 2009, p. 1-4. ISBN 978-184919-125-8.

[4] Demeter, E.; Faried, S.O.; Sidhu, T.S.; Signal phase shifting during synchrophasor measurements. Electrical and Computer Engineering, 2005. Canadian Conference on, 1-4 May 2005 Page(s):557 - 560. 